\title{
Repurposing metocurine as main protease inhibitor to develop novel antiviral therapy for COVID-19
}

\author{
Rashi Jain ${ }^{1} \cdot$ Somdutt Mujwar ${ }^{1}$ (D) \\ Received: 10 June 2020 / Accepted: 30 July 2020 / Published online: 11 August 2020 \\ (C) Springer Science+Business Media, LLC, part of Springer Nature 2020
}

\begin{abstract}
The outbreak of severe acute respiratory syndrome coronavirus-2 is causing a serious disaster through coronavirus disease-19 (COVID-19) around the globe. A large segment of the population from every corner of the world is already infected with this dreadful pathogen with a high mortality rate. These numbers are increasing drastically causing a situation of a global pandemic. Although after the continuous scientific efforts, we are still not having any specific drug or vaccine for the SARS-CoV-2 pathogen to date and there is an urgent need to develop a newer therapy to counter the COVID-19 global pandemic. Thus, in the current study, a framework for computational drug repurposing is established, and based on their safety profile, metocurine was chosen as a safe and effective drug candidate for developing therapy against the viral Mpro enzyme of SARS-CoV-2 for the treatment of COVID-19.
\end{abstract}

Keywords Drug repurposing $\cdot$ Drug repositioning $\cdot$ SARS-CoV-2 $\cdot$ COVID-19 $\cdot$ Corona $\cdot$ Antiviral

\section{Introduction}

Coronaviruses are spherically enveloped particles accommodating single-stranded positive-sense RNA combined with a nucleoprotein in a capsid constituted of matrix protein. These are typified by the club-like spikes extruding from its surface. The virus contains uncommonly large RNA genome with an exclusive replication approach. It includes respiratory symptoms such as fever, cough, and inadequacy in breathing. The disease is coined as COVID-19 which is escalated by a novel Betacoronavirus. Coronavirus responsible for the epidemic is now termed as severe acute respiratory syndrome coronavirus-2 (SARS-CoV-2), which has 79\% sequential similarity to SARS$\mathrm{CoV}$, causing the outbreak in 2002-2003. SARS-CoV-2 was inclusive of Middle East Respiratory Syndrome (MERS) coronavirus. [1-3].

Coronavirus is transmitted from person-to-person when an infected individual person coughs or sneezes. This virus can

Somdutt Mujwar

somduttmujwar@gmail.com

Rashi Jain

rashijainsjs@gmail.com

1 Institute of Pharmaceutical Research, GLA University, Mathura, Uttar Pradesh 281406, India also escalate via contaminated surfaces to hands and then to an individual. The transmission is usually via airborne droplets to the nasal mucosa, replicating narrowly in cells of ciliated epithelium leading to cell damage and inflammation $[4,5]$.

The virus enters the host cell, and uncoated genome transcribes and translates. The mRNAs assemble an onliest "nested set" allocating a common 3' end [6]. New virions are developed through budding from host cell membranes. The coronavirus leads to acute and chronic respiratory, enteric, and CNS diseases in various species of animals, including humans [2]. These viruses plunder the respiratory tract via the nose. The symptoms of common cold along with nasal obstruction, cough, running nose, sneezing, etc. are observed after the incubation period of about 3 days [7].

The marked organ that gets affected by $\mathrm{CO}-\mathrm{V}$ is extensively considered to be the lungs. Patients oftentimes have indication of else organ dysfunction, inclusive of GIT symptoms, lymphadenopathy, abnormal liver, and splenic atrophy. The five out of eight confirmed cases have revealed the apparent degenerative changes of hepatocytes in centrilobular zone and focal atrophy in testes of seven male patients. It is conspicuous that the infection by the virus is not restrained to the lungs and however implicates other parts of the respiratory tract and other organ systems too, uttermost particularly immune cells, notably the T lymphocytes, monocytes, and macrophages $[2,8]$. 
Fig. 1 Computational framework of drug repurposing to identify competitive inhibitors of viral Mpro enzyme to develop newer antiviral therapy against SARS-CoV-2

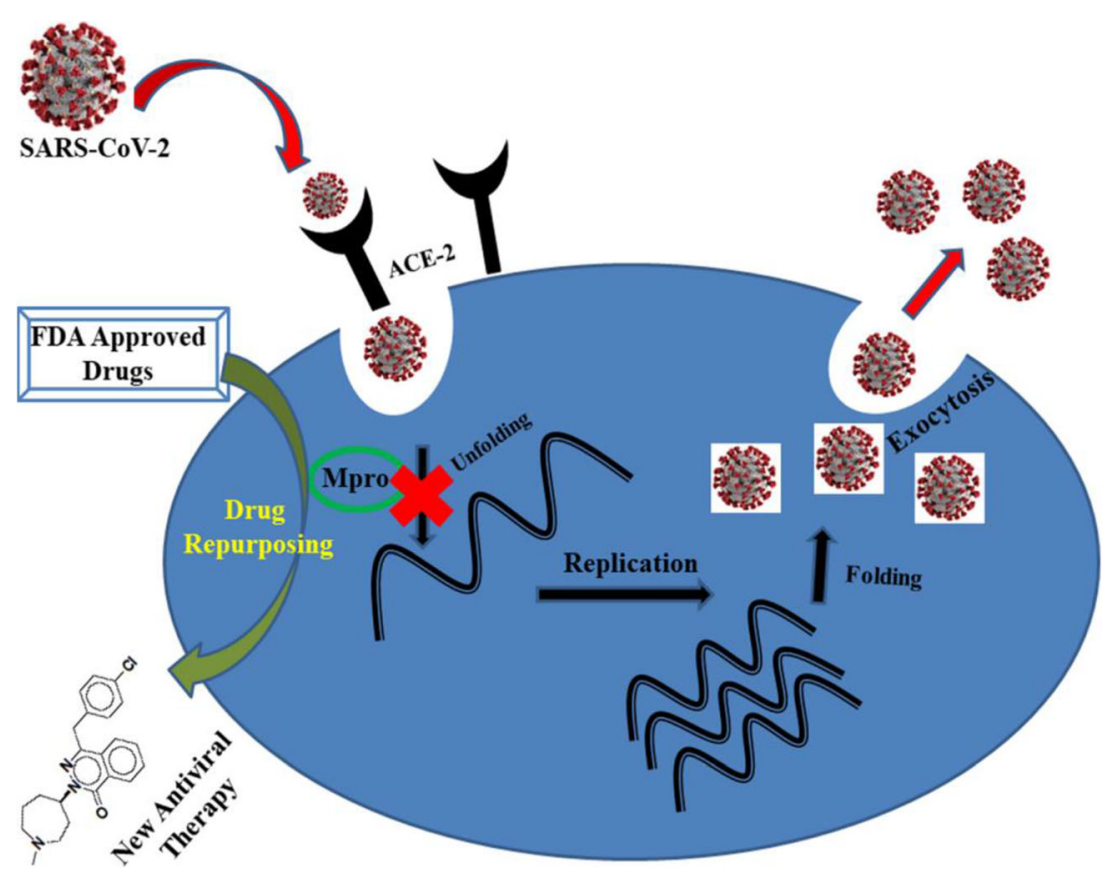

Globally, there are 1,288,525 confirmed cases of people affected with coronavirus. Out of which, the mortality rate has reached to the number of 70,570 in more than 200 countries, areas, or territories. According to the WHO, China is the first country reported for 81,708 confirmed cases for coronavirus with 3331 deaths. The maximum number of infected patients until date is observed in the USA with 336,907

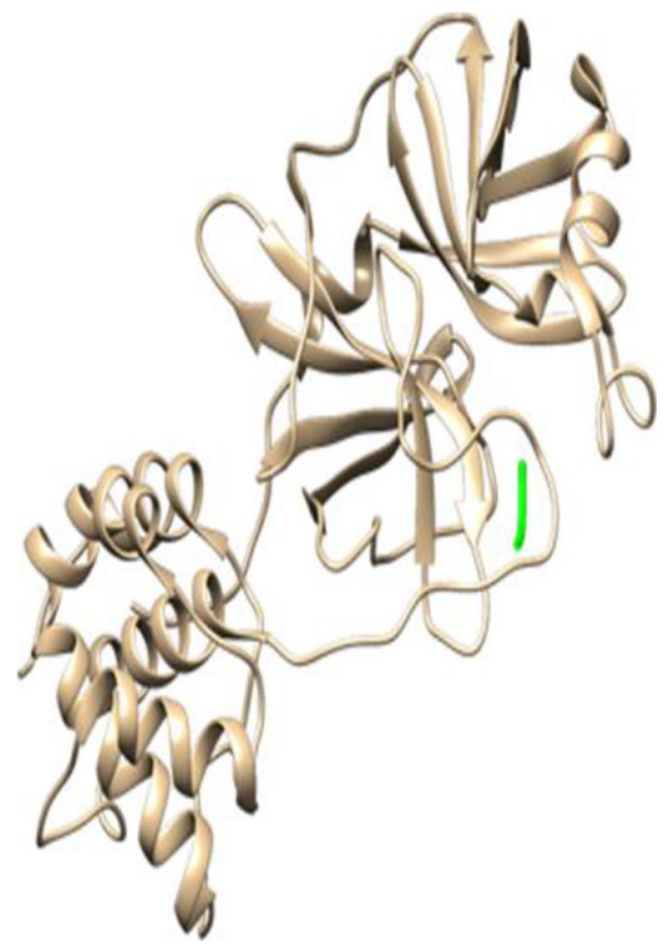

Fig. 2 Structural model of the viral main protease (Mpro) enzyme complexed with a peptide antagonist (PDB ID-6luz) confirmed cases with more than 10,000 deaths, and Italy and Spain come with more than 100,000 number of people confirmed in both the countries with the effect of coronavirus, and the mortality rate reaches up to 15,000 in each of the countries [7-9].

The viral main protease (Mpro) enzyme is found to be having a significant role in the entry of the pathogen into the host cell and can be targeted as a potential drug target. The inhibition of the viral Mpro enzyme will lead to the termination of the viral entry into the host cell resulting in the cessation of the viral infection.

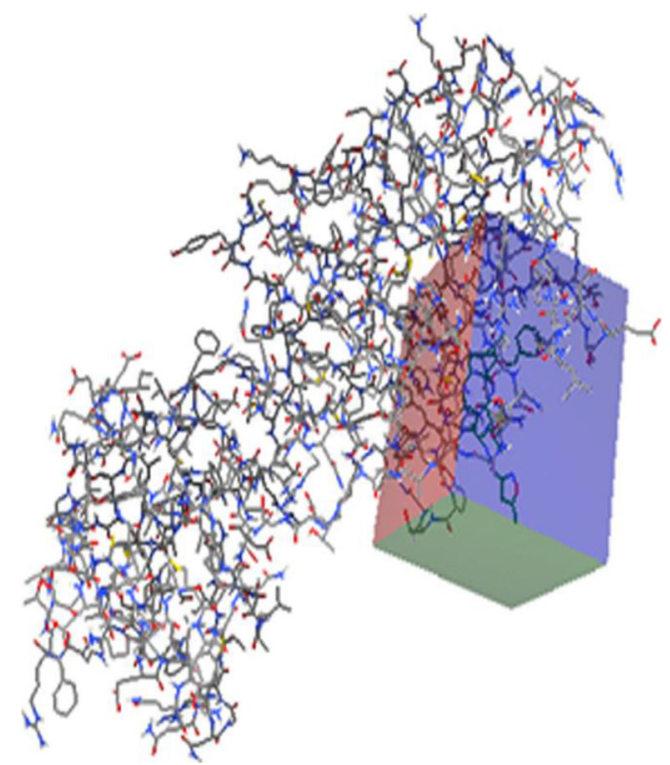

Fig. 3 3D grid-box utilized in the docking studies of the viral Mpro enzyme 
Fig. 4 The chemical interaction of the viral Mpro enzyme with the bound ligand $\mathrm{N} 3$

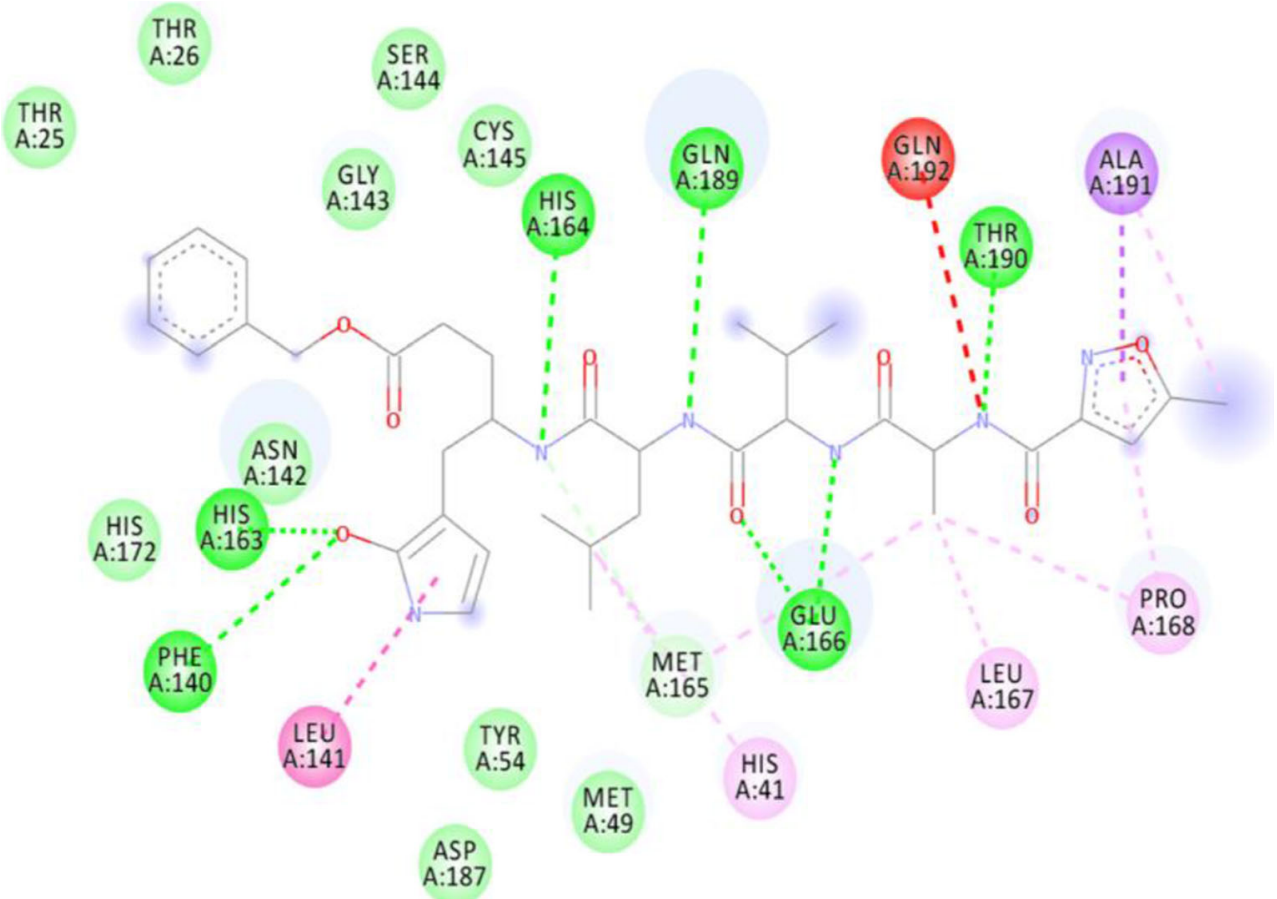

Thus, a potential viral Mpro enzyme inhibitor is supposed to be an effective therapy to counter the pandemic situation of COVID-19 around the globe [10, 11]. The graphical demonstration of the developed framework of computational drug repurposing to competitive inhibitors of viral Mpro enzyme is shown in Fig. 1.

Drug repurposing is a cost-effective advanced strategy for current diseases involving the rapid building of alternative therapies considering existing diseases. Drug repurposing methods are applied by implementing assorted in silico techniques like virtual screening of approved drug libraries and molecular docking simulation of new drug targets. In silico approaches are used to analyze, integrate, and administer large-scale information derived from scientific data. Computational drug repositioning adds a systematic and rational solution for perceiving treatment options correlated to conventional drugs $[12,13]$.

Thus, in the current study, molecular docking simulationbased computational drug repurposing approach is utilized to identify potential inhibitor of main protease (Mpro) enzyme of the severe acute respiratory syndrome coronavirus- 2 for developing novel therapy to counter coronavirus disease-19 (COVID-19).

\section{Experimental}

\section{Selection and preparation of macromolecule for docking}

The main protease (Mpro) enzyme of the severe acute respiratory syndrome coronavirus-2 (SARS-CoV-2) bound with a peptide-like inhibitor molecule N3 (PDB code-6luz) was obtained from the RCSB Protein Data Bank (PDB) $[10,14]$. The three-dimensional (3D) graphical representation of the viral Mpro was shown in Fig. 2. The ligand molecule was separated from the structural protein model of viral Mpro enzyme by using the software Chimera. The water molecules that are not having any interacting role between the macromolecular residues and the ligand were removed, and the polar hydrogen atoms were added to the macromolecular structure model for performing docking simulations $[15,16]$.

\section{Ligand preparation for docking}

The rotatable, unrotatable, and non-rotatable bonds in the peptide-like ligand N3 were endorsed in AutoDock for performing docking simulations [15].
Table 1 The coordinates of gridbox for the viral Mpro enzyme

\begin{tabular}{llllllll}
\hline Macromolecule & $x$-axis & $y$-axis & $z$-axis & Spacing $(\AA)$ & $x$-center & $y$-center & $z$-center \\
\hline 6lu7 & 40 & 54 & 40 & 0.419 & -9.732 & 11.403 & 68.925 \\
\hline
\end{tabular}


Table 2 Docking results of ligand N3 against the viral Mpro enzyme (6lu7)

\begin{tabular}{lllc}
\hline Macromolecule & Binding residue & RMSD & $\begin{array}{c}\text { Binding energy } \\
(\mathrm{kcal} / \mathrm{mol})\end{array}$ \\
\hline 6lu7 & $\begin{array}{c}\text { Ala191, Thr190, Glu166, Leu167, Leu141, } \\
\text { His163, Gln189, Met49, His41, His164, and Gly143 }\end{array}$ & 0.89 & -8.23 \\
\hline
\end{tabular}

\section{Binding site identification}

The binding site was identified within the structural model of viral Mpro enzyme by using the interacting residues of the macromolecule to the N3 ligand with the help of PyMOL software [13].

\section{Molecular docking}

The identified binding site within the viral Mpro enzyme was further utilized to specify the grid parameter points required to

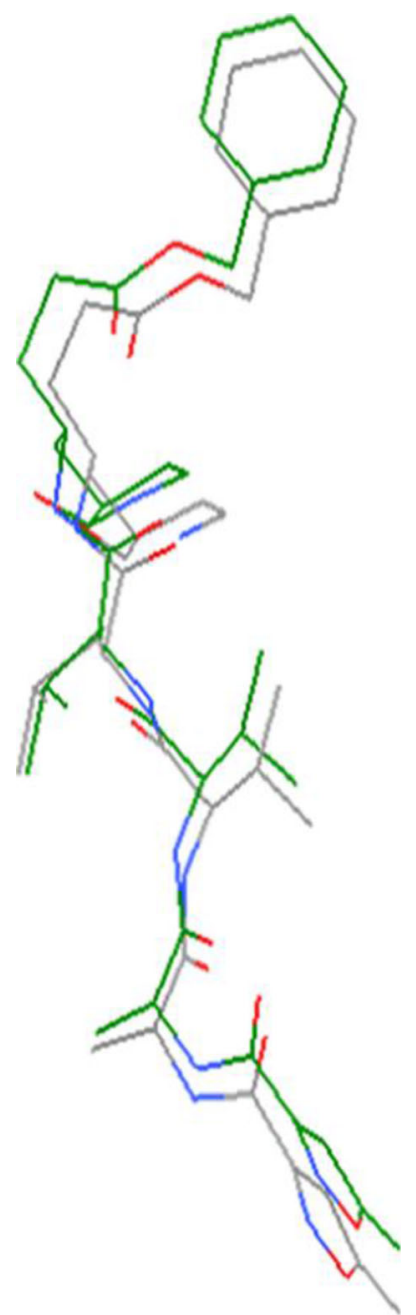

Fig. 5 The perfectly superimposed overlaid conformation of the docked ligand N3 with respect to its crystallized conformation obtained from the bioactive complex structure generate grid-box. The generated grid-box was further utilized to perform all the docking cycles performed in the current in silico studies. The grid-box was located by considering the ligand as a center and also wrapping each of the macromolecular residues interacting with the ligand to make sure that every possible legend conformation falls inside the grid-box [16]. The grid-box used in the current in silico studies was shown in Fig. 3.

The software AutoGrid generates map files for different types of atoms present in the ligand as well as receptor. In the current experimental study A C HD N OA SA NA, etc., map files are prepared by the AutoGrid. The prepared map files are utilized by AutoDock software for performing molecular docking simulations [15] [16].

The AutoDock software utilizes Lamarckian genetic algorithm (LGA) as its primary conformational search algorithm for performing the docking studies. LGA generates a trail population of a range of conformations of the ligand, followed by the generation of the mutational conformations and exchange of different parameters to contend in a style related to consecutive generations of the biological evolutions for the ultimate selection of the individuals having minimum binding energy. The additional feature of the "Lamarckian" aspect includes the individual and selective conformational search for their local conformational space followed by the identifying local minima. Later, the generated information is transferred to the later generations. The semi-empirical force field was utilized to predict the ligand's binding energy with reference to the specific macromolecular target. The force field predicts the binding energy of the ligand by allowing the integration of the intra-molecular energies by evaluating the energetics for their bound and unbound states on the basis of a comprehensive thermodynamic model. The docking parameter file (DPF) consists of various parameters required to perform docking of each ligand molecule [12, 17].

\section{Validation of docking methodology}

The ligand's probable binding pattern was obtained on the basis of their position and orientations identified after the molecular docking simulations. The parameters included in the current in silico study were validated by performing docking of the viral Mpro enzyme against the crystallized ligand N3. The crystallized conformation of the ligand N3 complexed within the active site of the viral Mpro enzyme is its bioactive 

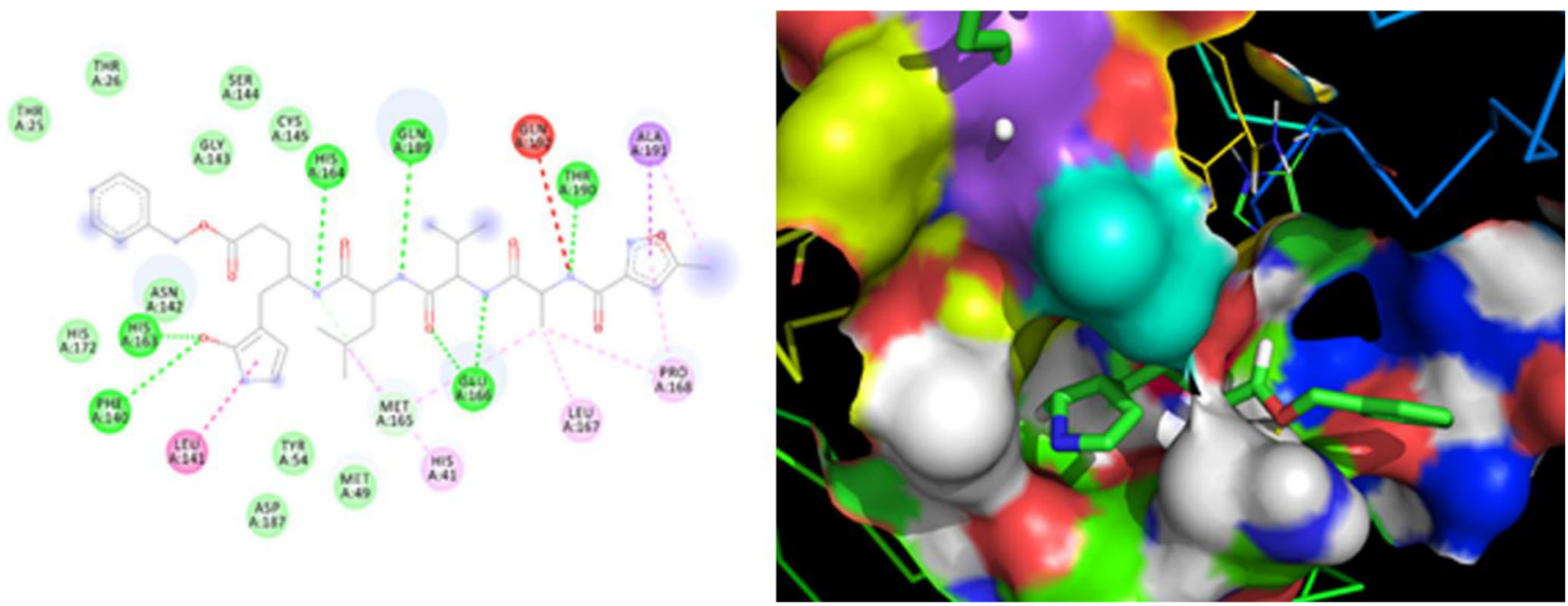

Fig. 6 Chemical interactions and the bound mode of the ligand N3 within the viral Mpro enzyme's binding site

conformation. The in silico docking methodology was validated through analyzing the subsequent parameters:

\section{(a) Overlay methods}

The ligand overlay methodology was utilized for the validation of docking parameters utilized in the current experimental study. The parameters utilized in the current docking study should be successfully validated if the docked conformation was immaculately overlaid over the crystallized bioactive conformation of the ligand available in the macromolecular complex.

\section{(b) Chemical resemblance}

The current docking methodology should be successfully validated if similar binding interactions are shown by the docked ligand with the macromolecular binding residues, as those were already present in the bioactive crystallized macromolecular complex structure.

The demonstration of the perfect overlay and similar binding interactions by the bound ligand $\mathrm{N} 3$ in its docked conformation with reference to its bioactive conformation should suggest that the molecular docking methodology is simulating the exact binding process of ligand within the receptor's active binding site as it occurs in the biological cellular system.

\section{In silico virtual screening}

The ligand library containing 2880 FDA-approved drugs was used in the current study to perform virtual screening against viral Mpro enzyme. The virtual screening of all these ligands was performed against a validated viral Mpro enzyme to identify potential lead molecules [16].

\section{Analysis of docking results}

The evaluation of the docking results for all the ligands against the viral Mpro enzyme was performed by considering the ligand's interactions with the macromolecular binding residues. The free binding energy for each of the ligand should be well within the predefined empirical range of -5 to - $15 \mathrm{kcal} / \mathrm{mol}$. The lead molecules were shortlisted by considering the lowest free binding energy. The LGA was utilized as a scoring function in the AutoDock tool. The following mathematical equation is used to generate the binding affinity of the specific ligand against as specified macromolecular target:

$K_{\mathrm{i}}=e^{[(\Delta \mathrm{G} /(\mathrm{RT})]}$

where $\Delta G=$ free energy change in binding, $T=$ temperature, and $R=$ gas constant.

\section{Results and discussion}

\section{Macromolecular selection and its preparation}

The viral Mpro enzyme complexed with ligand N3 (pdb code6lu7) was obtained from PDB database. Three-dimensional protein structural model was procured with the help of X-ray diffraction method at a resolution of $2.16 \AA$. The protein structure is having a single chain of 306 amino acid residues. The bound ligand $\mathrm{N} 3$ was removed from the protein complex (6lu7) by using the software Chimera. The molecular receptor was prepared to perform the docking experiment by removing the unwanted water molecules, adding up polar hydrogen, followed by addition and equal distribution of Gasteiger 
Table 3 Binding energy of shortlisted top 10 leads for the viral Mpro enzyme

\begin{tabular}{|c|c|c|c|c|}
\hline S. No. & Zinc Code & Name & Chemical Structure & $\begin{array}{c}\text { Binding } \\
\text { Energy }\end{array}$ \\
\hline 1 & ZINC04097448 & Metocurine & & -10.51 \\
\hline 2 & ZINC14880002 & Dihydroergotoxine & & -10.14 \\
\hline 3 & ZINC19632618 & Imatinib & & -10.12 \\
\hline 4 & ZINC11592928 & Daunorubicin & & -10.02 \\
\hline 5 & ZINC53683151 & Bromocriptine & & -9.95 \\
\hline 6 & ZINC01612996 & Irinotecan & & -9.63 \\
\hline 7 & ZINC00601229 & Azelastine & & -9.48 \\
\hline 8 & ZINC03814394 & Gestodene & & -9.39 \\
\hline 9 & ZINC03954487 & Adapalene & & -9.29 \\
\hline 10 & ZINC03831450 & Simvastatin & & -9.21 \\
\hline
\end{tabular}



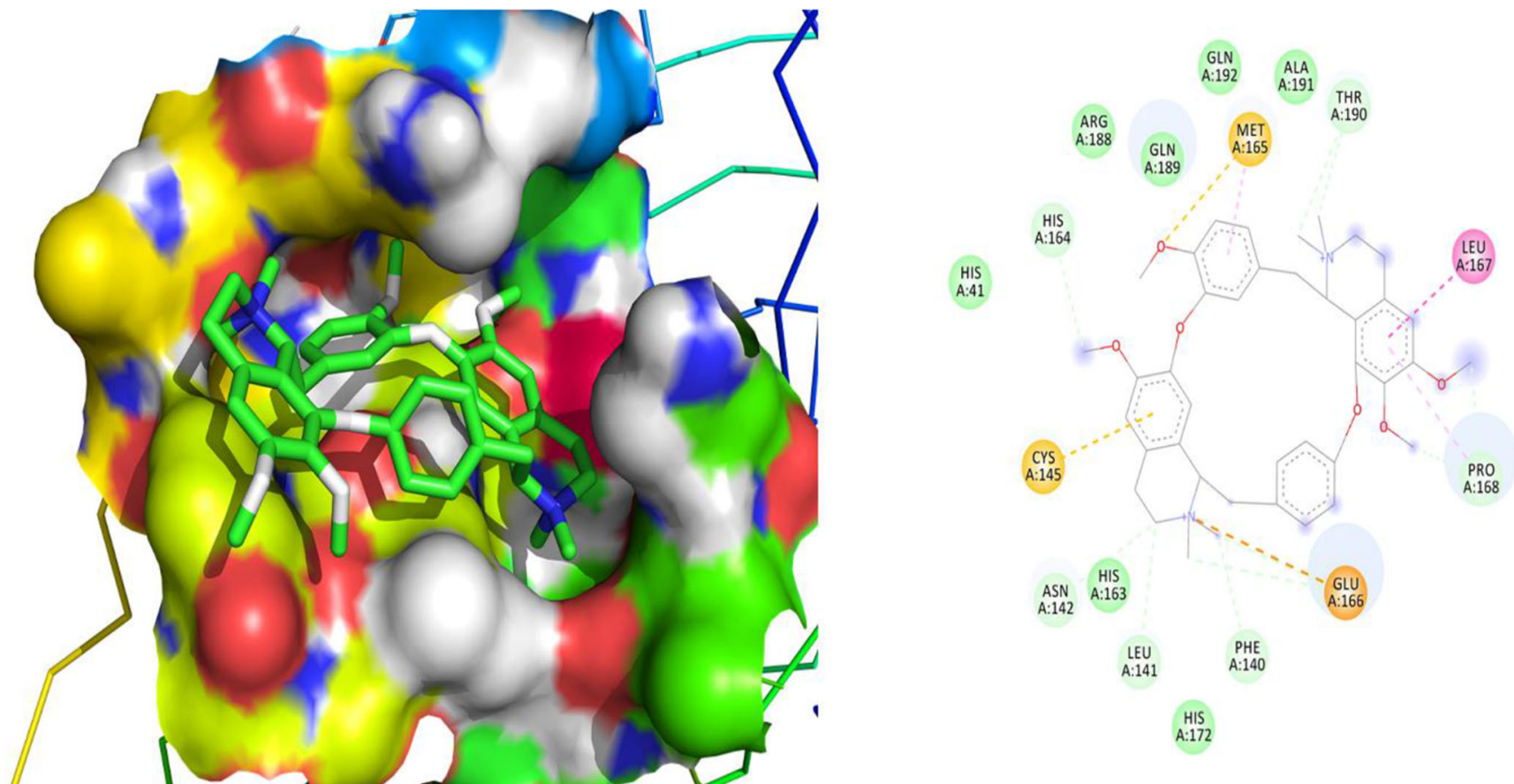

Fig. 7 Two- and three-dimensional binding interactions of metocurine: the binding interactions of metocurine with the viral Mpro

charge. The processed structural model of the protein was saved in the .pdbqt format of the AutoDock software.

\section{Ligand's preparation}

Nine aromatic carbons having 26 rotatable bonds are present in the bound ligand N3. All the 26 bonds of the N3 ligand were kept rotatable in the current computational study, and it was also saved in the .pdbqt format of the AutoDock software.

\section{Ligand binding site identification and grid-box formation}

The macromolecular binding residues Ala191, Thr190, Glu166, Leu167, Leu141, His163, Gln189, Met49, His41, His 164, and Gly143 were having interacting role in the active binding of the ligand N3 with the viral Mpro enzyme. The chemical interactions of the ligand N3 with the viral Mpro enzyme were shown in Fig. 4.
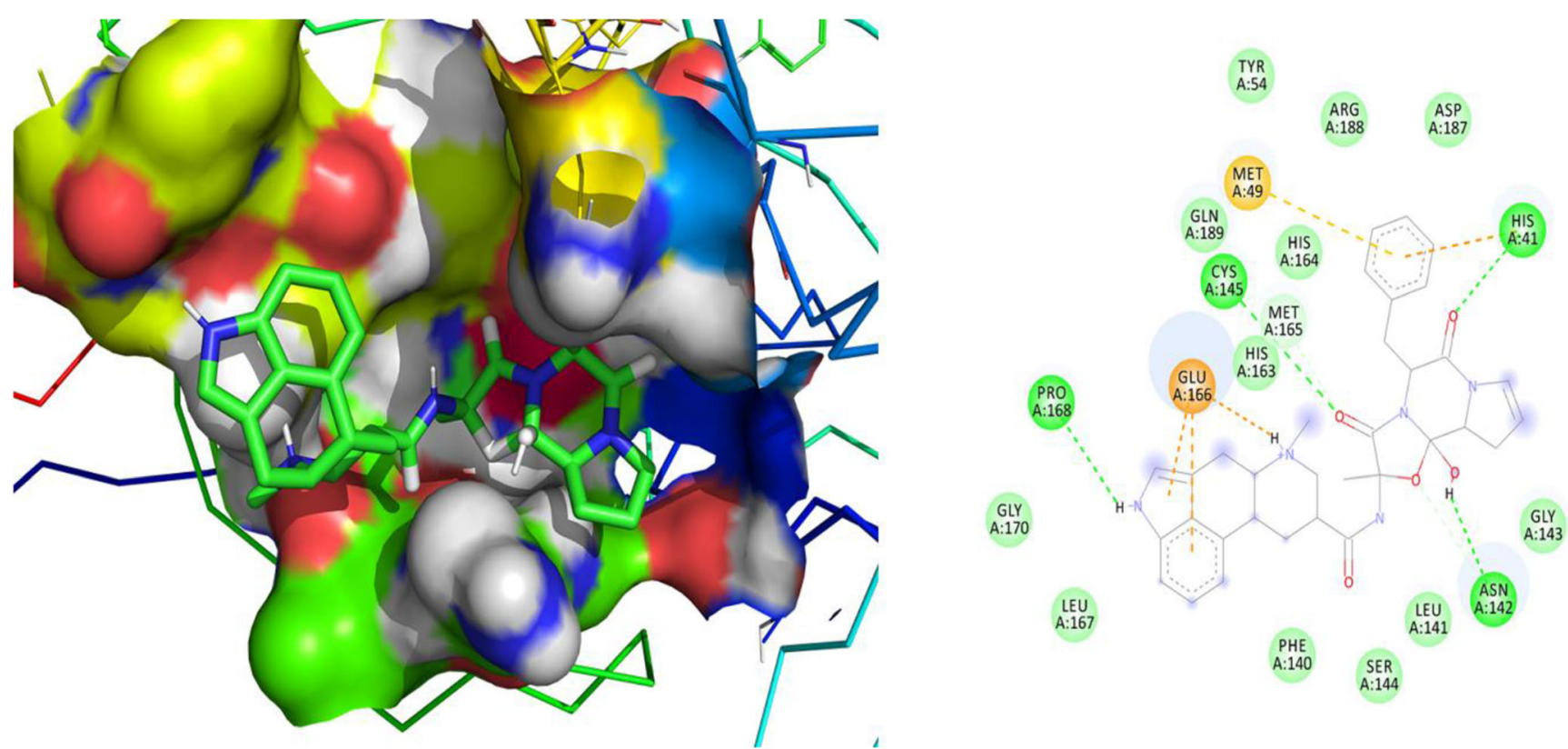

Fig. 8 Two- and three-dimensional binding interactions of dihydroergotoxine: the binding interactions of dihydroergotoxine with the viral Mpro 


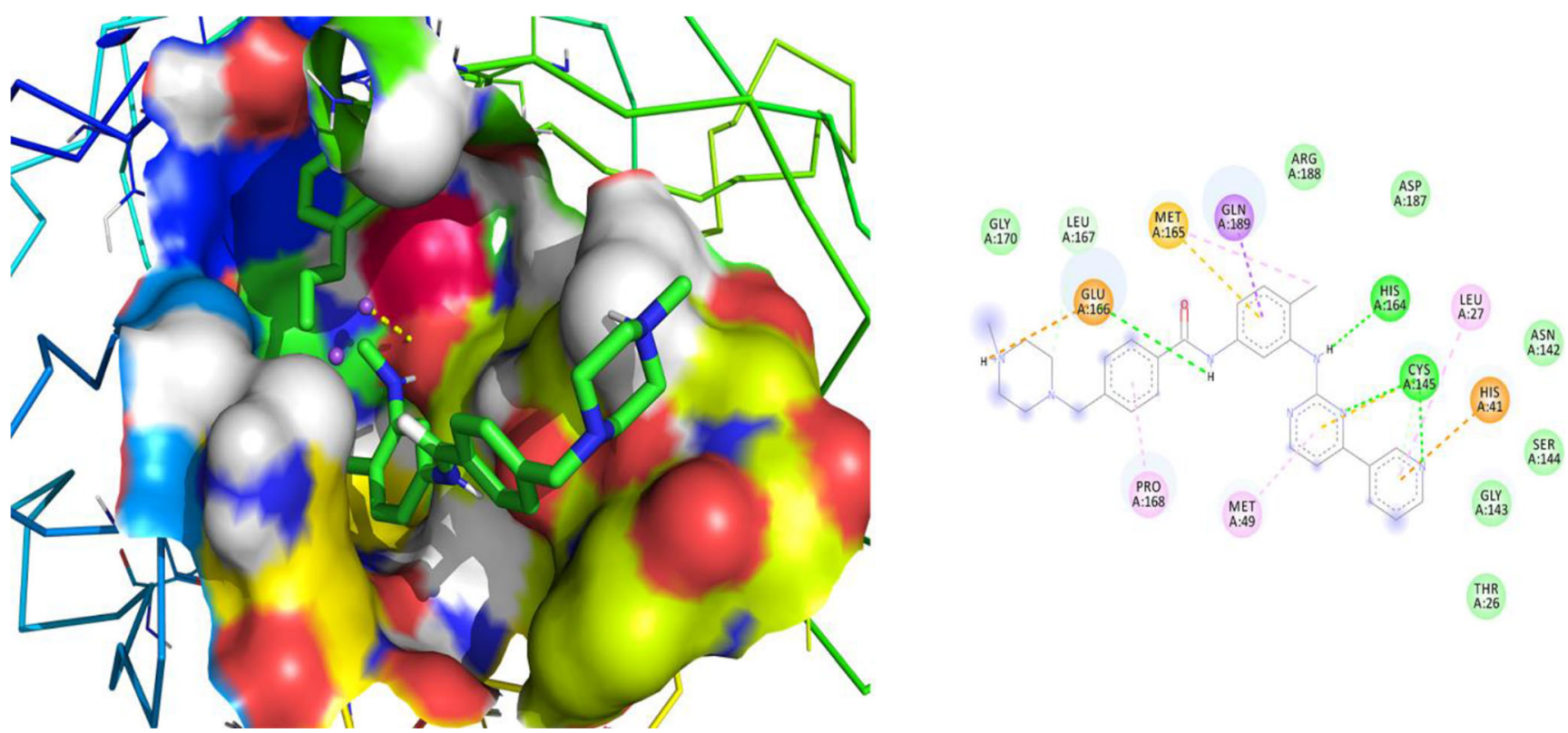

Fig. 9 Two- and three-dimensional binding interactions of imatinib: the binding interactions of imatinib with the viral Mpro

An imaginary grid-box was arranged by wrapping up the ligand molecule $\mathrm{N} 3$ as well as all the binding residues of the viral Mpro enzyme. The grid coordinates utilized for the preparation of the grid-box were given in Table 1.

\section{Validation of molecular docking}

The docking results of the bound ligand N3 against the viral Mpro enzyme were shown in Table 2.
The method validation for the docking process for performing the molecular docking of the viral Mpro enzyme was performed by applying the following parameters:

(a) Overlay method

The docked conformation of the ligand N3 was perfectly overlaid over its docked conformation; thus, the molecular docking methodology was successfully validated for the current in silico studies.
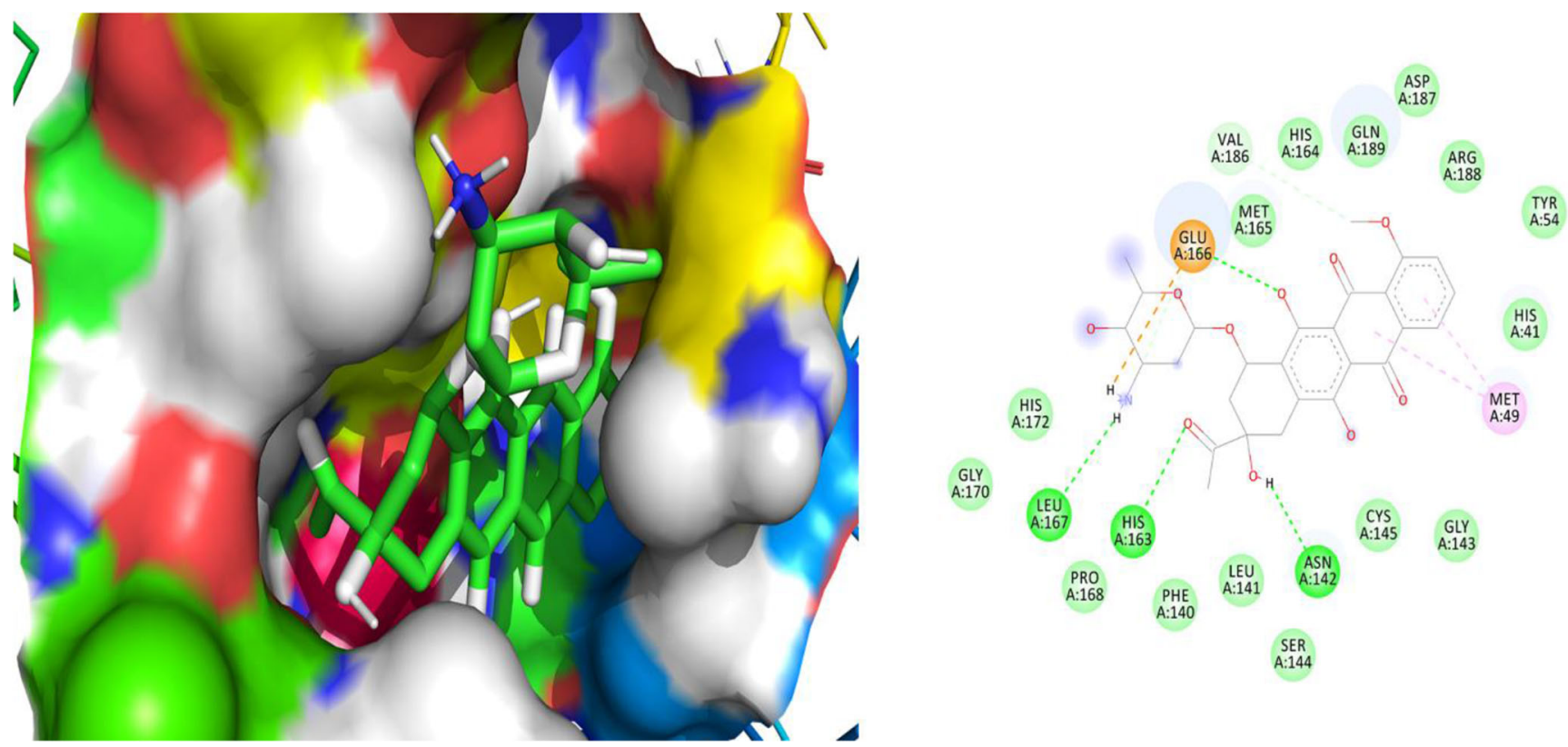

Fig. 10 Two- and three-dimensional binding interactions of daunorubicin: the binding interactions of daunorubicin with the viral Mpro 

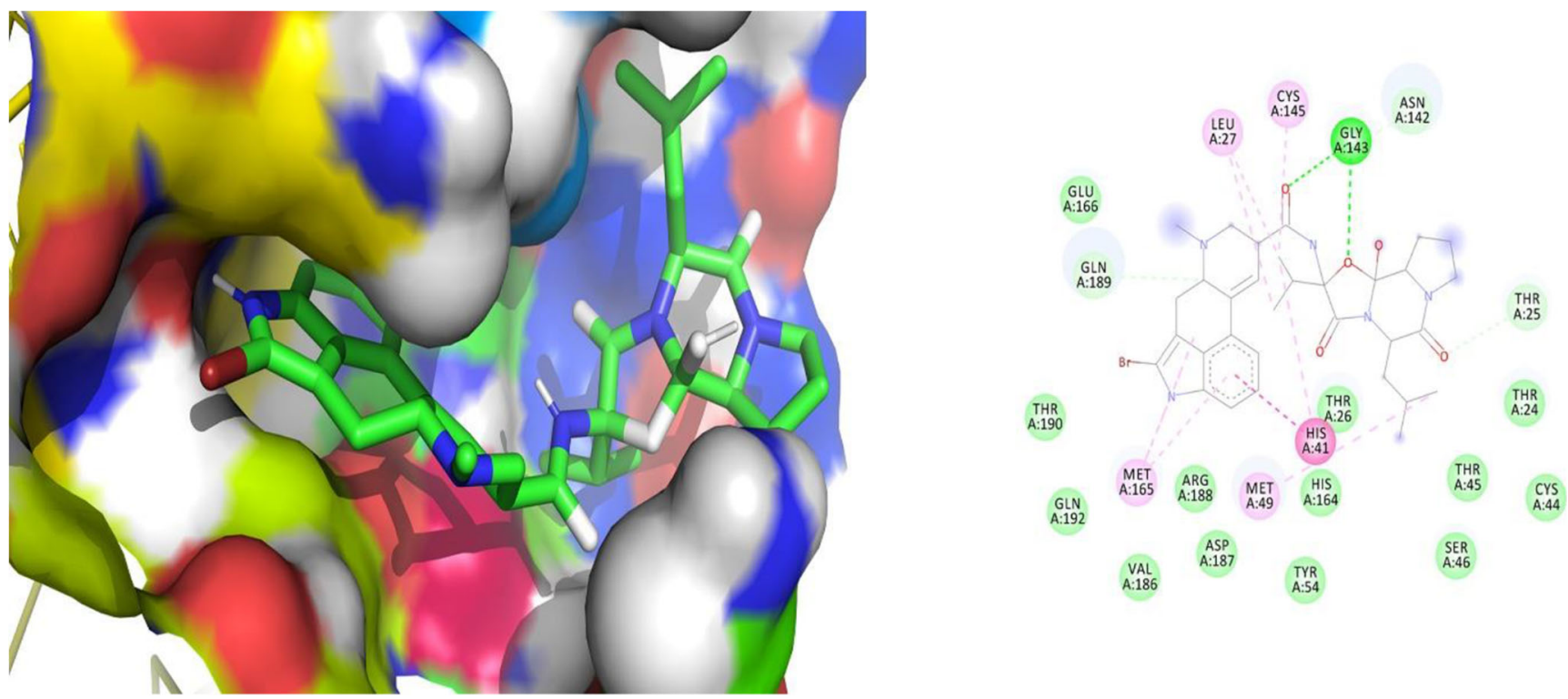

Fig. 11 Two- and three-dimensional binding interactions of bromocriptine: the binding interactions of bromocriptine with the viral Mpro

The perfectly overlaid conformation of the docked ligand N3 with respect to its crystallized conformation was presented in Fig. 5.

\section{(b) Chemical resemblance}

The current docking process of the viral Mpro enzyme was further validated as the docked ligand is having the similar chemical interactions with macromolecular residues as those were shown within its bioactive crystallized structure. The binding interactions of both docked and the crystallized structure were presented in Fig. 6.

The exact overlay as well as similar binding interactions of the docked conformation of the ligand $\mathrm{N} 3$ with respect to its bioactive conformation obtained from the crystallized macromolecular complex successfully validates that the molecular docking methodology is exactly simulating the similar complexation of the ligand and the viral macromolecular assembly as it is happening within the biological cellular assembly.

\section{Virtual screening}

The leads were selected on the basis of their affinity against the viral Mpro enzyme. The binding affinity of the leads was evaluated by considering lower binding energy acquired for preeminent pose for every ligand and their binding interactions with the macromolecule. The binding energy obtained

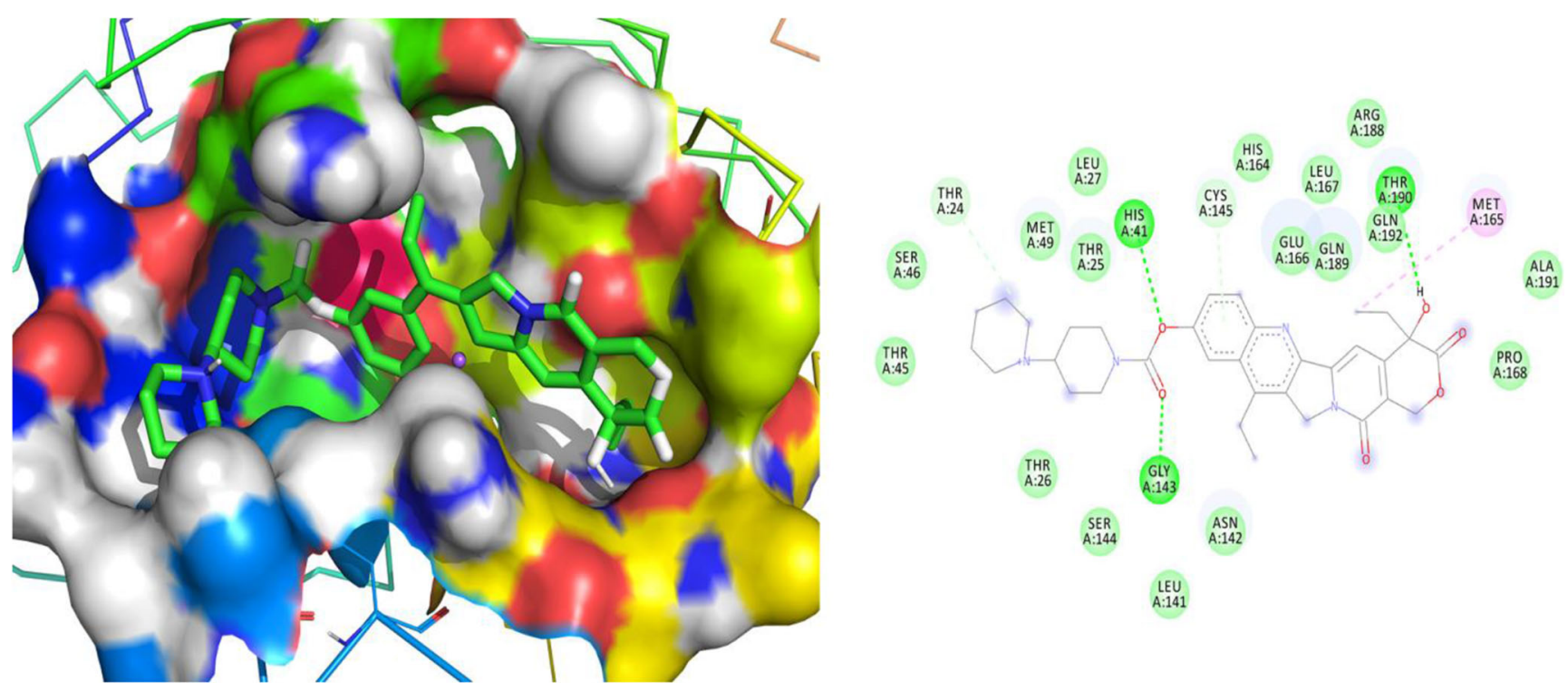

Fig. 12 Two- and three-dimensional binding interactions of irinotecan: the binding interactions of irinotecan with the viral Mpro 

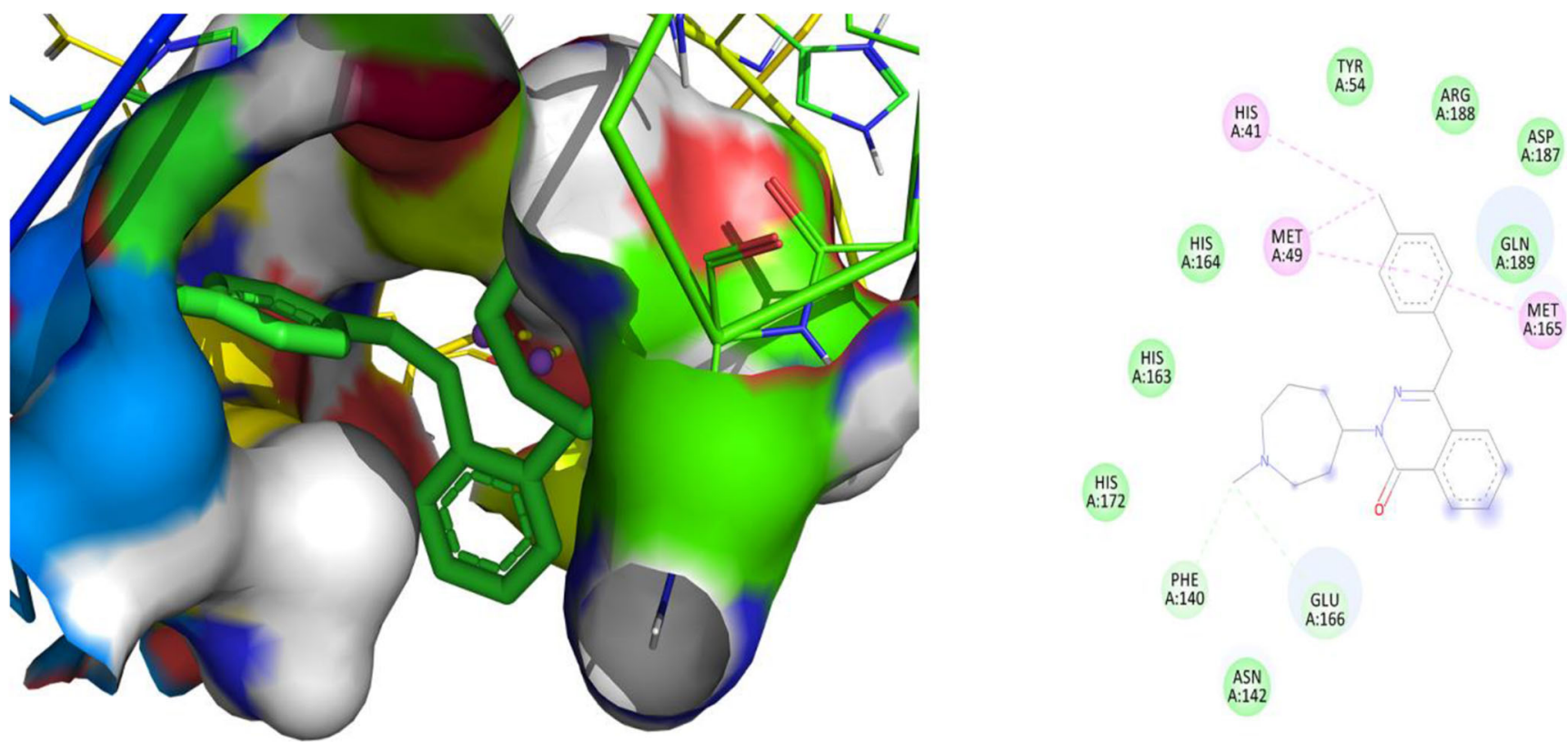

Fig. 13 Two- and three-dimensional binding interactions of azelastine: the binding interactions of azelastine with the viral Mpro

after docking-based virtual screening of the top 10 shortlisted ligand molecules was shown in Table 3.

\section{Discussion}

The capability of rapid multiplication and contagious nature of the SARS-CoV-2 are responsible for causing the COVID-19 pandemic situation around the globe. The COVID-19 disaster has further increased because of the lack of any specific therapy against the viral pathogen. Discovery of some selective and diverse pathogenic drug targets is highly favorable to quickly identify new clinically active drugs. The viral Mpro enzyme is found to be having a significant role in the entry of the pathogen into the host cell and can be targeted as a potential drug target. Therefore, computational screening of the clinically approved drugs would be highly propitious for rapid development of newer therapy against the infectious COVID19 disease affecting mankind [11].

The drug development process is a very complex process which desires so many years as well as large approach of money for discovery of new chemical entities (NCEs). To overcome the high cost and time required for developing a drug molecule, in silico drug repurposing and repositioning techniques are promising alternative methods. In the current paradigm of conventional drug discovery process, the drug repurposing approach reveals an alternative economical and time-saving substitute having a higher success rate. Drug
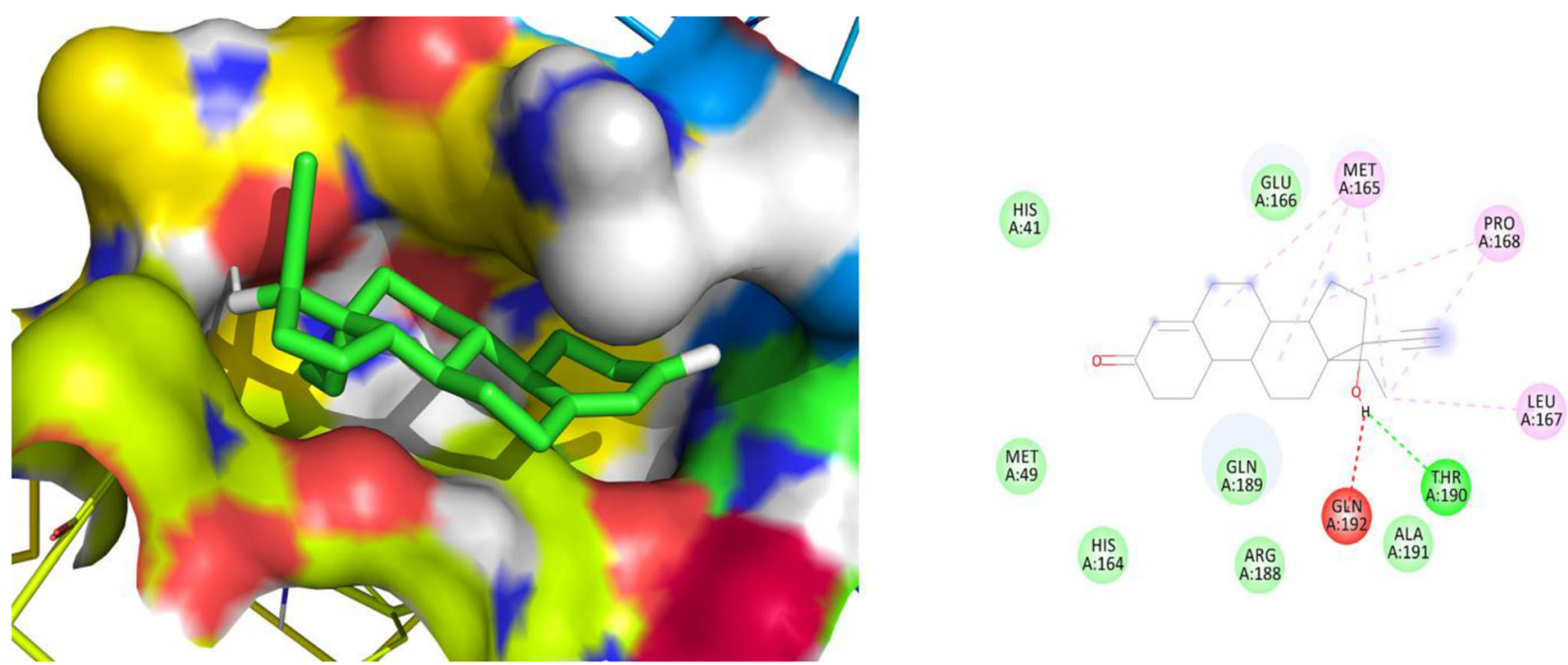

Fig. 14 Two- and three-dimensional binding interactions of gestodene: the binding interactions of gestodene with the viral Mpro 

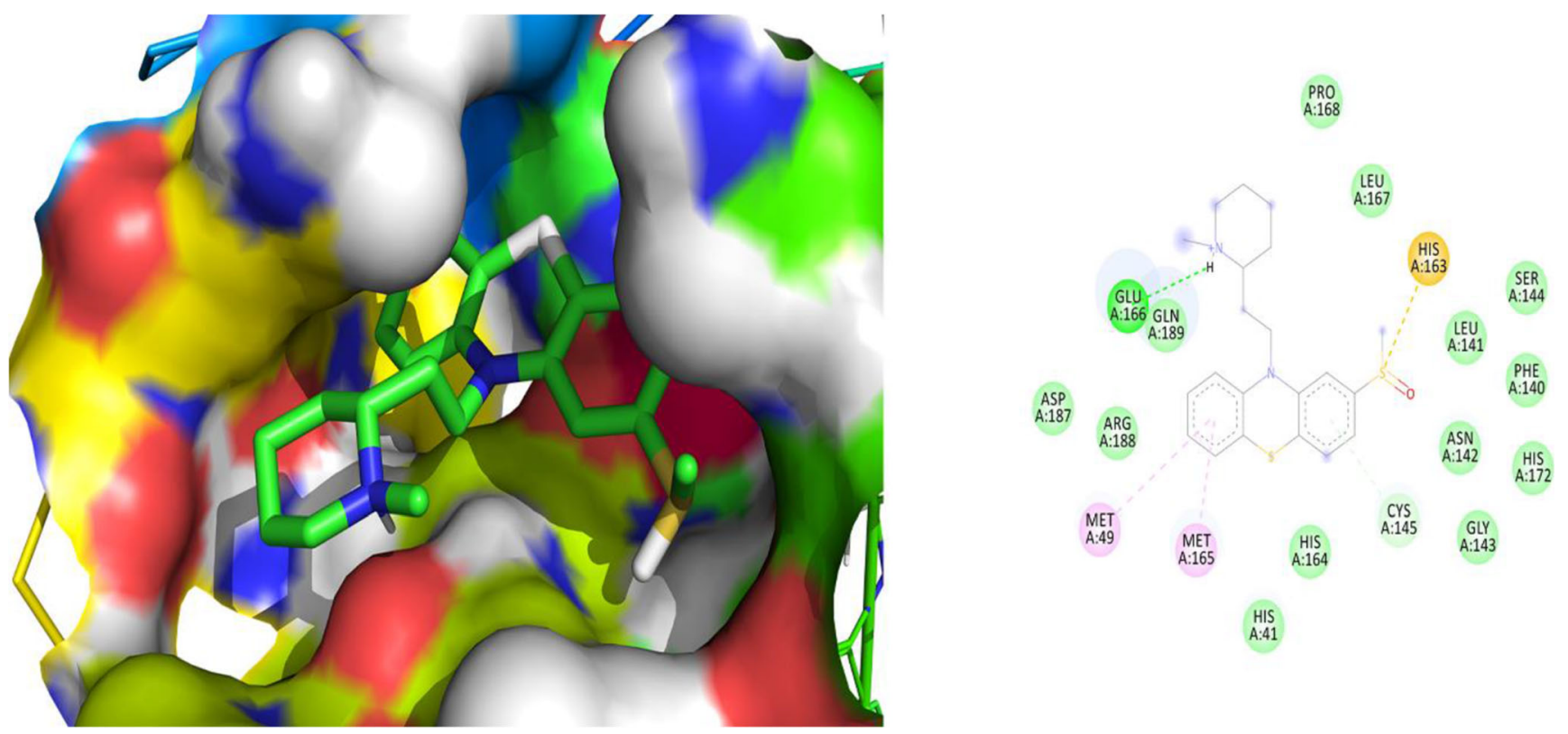

Fig. 15 Two- and three-dimensional binding interactions of adapalene: the binding interactions of adapalene with the viral Mpro

repurposing is a technique in which a newer pharmacological application of an existing drug is identified and established through computational and clinical investigations. Drug repurposing and repositioning techniques are extensively applied in the modern era for the successful development of newer therapy for the diseased condition via existing drugs. In the earlier days, the newer pharmacological role of an existing drug was identified by its accidental use or either observed by their clinical manifestations. Nowadays, fast, economical, reliable, and versatile computational repurposing techniques were utilized to recognize a newer functional role of an existing drug molecule.

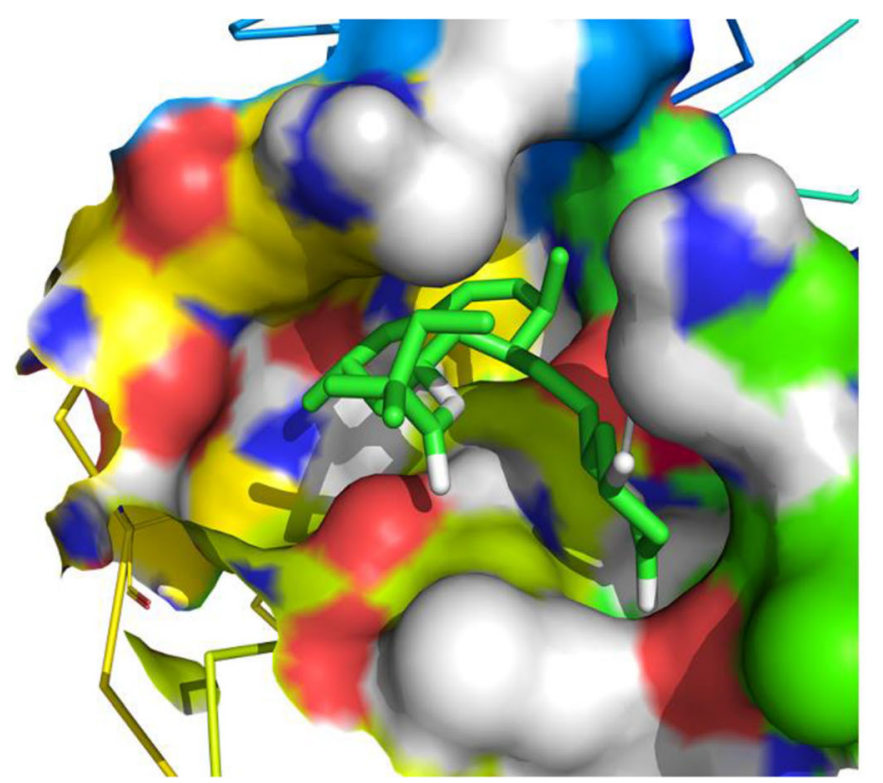

Drug repurposing is a contributing approach which is nowadays used for the identification of a novel pharmacological action of an existing drug molecule. In this context, in silico drug repurposing approach is utilized in the current biochemical standpoint to develop a novel therapy against the SARSCoV-2 viral strain by targeting the Mpro enzyme.

In the current biochemical standpoint, the threedimensional structure model of viral Mpro enzyme complexed with N3 is obtained from the RCSB protein data bank. The bound drug N3 is separated from the complex enzyme with the help of the Chimera software. The separated drug molecule is docked in the active binding site of the target protein by using AutoDock software for validating the

Fig. 16 Two- and three-dimensional binding interactions of simvastatin: the binding interactions of simvastatin with the viral Mpro 
parameters utilized in the molecular docking process. The findings of the current in silico virtual screening methods lead to the development of drug molecules like metocurine, dihydroergotoxine, imatinib, daunorubicin, bromocriptine, irinotecan, azelastine, gestodene, adapalene, and simvastatin against the target Mpro enzyme of SARS-CoV-2.

Metocurine is a competitive non-depolarizing neuromuscular blocking agent used as a muscle relaxant as well as an anesthetic agent. The metocurine interacts within the active binding cavity of viral Mpro enzyme by mainly with the Phe140, Leu141, Cys145, His163, His164, Met165, Glu166, Leu167, and Pro168 residues. Dihydroergotoxine is a neuroprotective agent and a nootropic agent whose mechanism is not clear yet. His41, Met49, Asn142, Cys145, Glu166, and Pro168 of the viral Mpro enzyme are found to be interacting with the dihydroergotoxine. Imatinib is kinase inhibitor used to treat certain types of cancer. Imatinib is found to be interacting with the Leu27, His41, Met49, Cys145, His164, Met165, Glu166, Pro168, and Gln189 residues of the viral Mpro enzyme. Daunorubicin is an anticancer agent which strongly interacts with the Met49, Asn142, His163, Leu167, Glu166, and Val186 residues of viral Mpro enzyme. Bromocriptine is a dopamine agonist that is used in the treatment of tumors, Parkinson's disease, and type 2 diabetes. The binding residues His41, Thr45, Gly143, Cys145, and His164 of viral Mpro enzyme are found to have interactions with bromocriptine. Irinotecan is an antineoplastic agent used for the treatments of colorectal and pancreatic neoplasms by interfering nucleic acid synthesis at S-phase by inhibiting DNA topoisomerase-I. The binding residues His41, Gly143, Met165, and Thr190 of viral Mpro enzyme are found to have interactions with irinotecan. Azelastine is an antihistaminic agent used in the treatment of allergies and rhinitis. Azelastine interacts with the residues like His41, Met49, Phe140, Met165, and Glu166 of the viral Mpro enzyme. Gestodene is a progestogen hormonal contraceptive which interacts with Met165, Leu167, Pro168, and Thr190 residues of the viral Mpro enzyme. Adapalene is anti-acne agent belonging to the class of the retinoid drug which interacts with Met49, His163, and Glu166 residues of the viral Mpro enzyme. Simvastatin is a synthetically derived lipid-lowering drug having interactions with the His41, Met49, Asn142, Met165, Glu166, and His172 residues of the viral Mpro enzyme. The binding interactions of all the shortlisted lead molecules were shown in Figs. 7, 8, 9, 10, 11, $12,13,14,15$, and 16 .

The structure activity relationship of the shortlisted lead molecules revealed on the basis of the in silico binding pattern with the viral Mpro enzyme clearly suggests that the residues like Glu166, Met165, Met49, His41, and Asn142 play an important role in the binding of the drug molecule with the viral Mpro enzyme and revealed to play an important role in the inhibition of viral Mpro enzyme for possessing antiviral activity.
The repurposing techniques are highly promising for reproducing the new biological role of existing drugs. The identified bioactive molecules may be useful for developing novel therapies against the COVID-19 caused by the SARS-CoV-2 in the near future. These drugs should be further explored through preclinical and clinical studies for validating the proposed hypothesis in the current biochemical standpoint.

\section{Conclusion}

In silico drug repurposing technique is a highly adequate, prudent, and quick approach for identifying an extant drug molecule having therapeutic activity against the viral Mpro enzyme of COVID-19. A ligand library containing 2880 drug molecules authorized by the FDA was virtually screened against the viral Mpro enzyme of COVID-19. It was observed that metocurine, dihydroergotoxine, imatinib, daunorubicin, bromocriptine, irinotecan, azelastine, gestodene, adapalene, and simvastatin were found to be potential lead molecules against the viral Mpro enzyme of COVID-19. Based on their safety profile, metocurine was chosen as a safe and effective drug candidate for developing therapy against the viral Mpro enzyme of SARS-CoV-2 for the treatment of COVID-19.

Acknowledgments The authors convey their heartfelt affection to the parents and family for their support to accomplish the research in the welfare of mankind. The authors are also thankful to the management of the GLA University, Mathura, for providing all the necessary facilities to complete the work.

\section{Compliance with ethical standards}

Conflict of interest The authors declare that they have no conflict of interest.

Consent for publication Not applicable.

\section{References}

1. Lake MA (2020) What we know so far: COVID-19 current clinical knowledge and research. Clin Med 20(2):124-127

2. Velavan TP, Meyer CG (2020) The COVID-19 epidemic. Trop Med Int Health 25(3):278-280

3. Lai CC, Shih TP, Ko WC, Tang HJ, Hsueh PR (2020) Severe acute respiratory syndrome coronavirus 2 (SARS-CoV-2) and coronavirus disease-2019 (COVID-19): the epidemic and the challenges. Int J Antimicrob Agents 55(3):1-9

4. Bai Y, Yao L, Wei T, Tian F, Jin D-Y, Chen L, Wang MJJ (2020) Presumed asymptomatic carrier transmission of COVID-19

5. Jernigan DB (2020) Update: public health response to the coronavirus disease 2019 outbreak-United States, February 24, 2020. MMWR. Morbidity and mortality weekly report 69(8):216-219

6. Tyrrell DA, Myint SH (1996) Coronaviruses. In Medical Microbiology. 4th edition. University of Texas Medical Branch at 
Galveston. Chapter 60. Available from: https://www.ncbi.nlm.nih. gov/books/NBK7782/

7. World Health Organization (2020) Coronavirus disease (COVID2019) situation reports. 2020. Available on: https://www.WHO.Int/ docs/defaultsource/coronaviruse/situationreports/20200221-sitrep32-covid, 19

8. Gu J, Gong E, Zhang B, Zheng J, Gao Z, Zhong Y, Zou W, Zhan J, Wang S, Xie Z et al (2005) Multiple organ infection and the pathogenesis of SARS. J Exp Med 202(3):415-424

9. Liu Y, Gayle AA, Wilder-Smith A, Rocklöv J (2020) The reproductive number of COVID-19 is higher compared to SARS coronavirus. J Travel Med 27(2)

10. Jin Z, Du X, Xu Y, Deng Y, Liu M, Zhao Y, Zhang B, Li X, Zhang L, Peng C (2020) Structure of Mpro from COVID-19 virus and discovery of its inhibitors. Nature 582:289-293

11. Hoffmann M, Kleine-Weber H, Schroeder S, Krüger N, Herrler T, Erichsen S, Schiergens TS, Herrler G, Wu N-H, Nitsche AJC (2020) SARS-CoV-2 cell entry depends on ACE2 and TMPRSS2 and is blocked by a clinically proven protease inhibitor

12. Mujwar S, Deshmukh R, Harwansh RK, Gupta JK, Gour A (2019) Drug repurposing approach for developing novel therapy against mupirocin-resistant Staphylococcus aureus. Assay Drug Dev Technol 17(7):298-309. https://doi.org/10.1089/adt.2019.944
13. Shah K, Mujwar S, Gupta JK, Shrivastava SK, Mishra P (2019) Molecular docking and in silico cogitation validate mefenamic acid prodrugs as human cyclooxygenase-2 inhibitor. Assay Drug Dev Technol 17(6):285-291. https://doi.org/10.1089/adt.2019.943

14. Berman HM, Westbrook J, Feng Z, Gilliland G, Bhat TN, Weissig H, Shindyalov IN, Bourne PE (2000) The Protein Data Bank. Nucleic Acids Res 28(1):235-242. https://doi.org/10.1093/nar/28. 1.235

15. Mujwar S, Pardasani KR (2015) Prediction of riboswitch as a potential drug target and design of its optimal inhibitors for Mycobacterium tuberculosis. 8(4):326-347

16. Mujwar S, Pardasani K (2015) Prediction of Riboswitch as a potential drug target for infectious diseases: an in silico case study of anthrax. J Med Imaging Health Infor 5(1):7-16

17. Minaz N, Razdan R, Hammock BD, Mujwar S, Goswami SK (2019) Impact of diabetes on male sexual function in streptozotocin-induced diabetic rats: protective role of soluble epoxide hydrolase inhibitor. Biomed Pharmacother 115:108897. https://doi.org/10.1016/j.biopha.2019.108897

Publisher's note Springer Nature remains neutral with regard to jurisdictional claims in published maps and institutional affiliations. 